\title{
ETHICS 2.0: AN INTROSPECTIVE APPROACH TO UNDERSTANDING AND TAKING OWNERSHIP OF YOUR ACTIONS
}

\author{
Karim S. Karim \\ Department of Electrical and Computer Engineering, University of Waterloo, Waterloo, Canada N2L3G1 \\ kkarim@uwaterloo.ca
}

\begin{abstract}
ECE290 (Engineering Profession, Law and Ethics) is a second year core course in the undergraduate Electrical and Computer Engineering program at the University of Waterloo. This course was designed to move away from achieving desired ethical outcomes or "right" answers and instead to focus on refining individual decision-making processes. Ethics was framed as stemming from the fundamental identity question faced by each individual and the core beliefs held by the individual with an aim to make the course more personal, engaging and introspective for the student. Students also gained practical experience in deconstructing their own identity by identifying and understanding master behavioral patterns and the perspectives of various characters in pertinent literature and case studies where ethical ambiguity is at the forefront. Evaluation and testing methods for the ethics component of the course were designed to focus on evaluating the depth and breadth of the students' decision-making processes. Student course evaluation questionnaires had a response rate of between 60-75\% and indicated that students strongly believed they were being encouraged to think critically and reason independently.
\end{abstract}

Keywords: Ethics, engineering, philosophy

\section{INTRODUCTION}

Approaches to teaching ethics in engineering at universities have typically focused on establishing an ethical foundation based on popular philosophical theories followed by practical discipline specific case analyses, and supplemented by in-class debates [1]. This approach has been prevalent not just in engineering departments but also in the professional schools of law, business, and medicine. Often, there is substantial discussion as to who is best qualified to teach courses on ethics.

With the constant daily barrage of media reports of professional ethical misdoings over the past decades, literally all universities have included an "ethics" course or component in their various professional program curricula although it is unclear whether any of these attempts are working. It is telling that even as early as Watergate [2], law schools were seeking an ethics renaissance.

The real challenge with teaching ethics is the seeming impossibility of "teaching" integrity because the traditional approach conflates ethics with morals and moralizing by an instructor is more so now, than it was ever in the past, counterproductive and ineffective. In fact, students often retain a belief that there is a right answer for the ethics course and a different answer for the real world where competing factors (e.g. employment security) hold greater sway.

Moreover, it can also be argued that efforts to "teach" a set of given values or any kind of basic ethical principles to university students are doomed to failure because of the myriad of perspectives on any given topic especially now since universities attract a more diverse student demographic than in the past. Also, learning in a free society cannot willingly force an outcome even if perceived as desirable by a set of stakeholders.

In the past, virtues such as integrity were developed through various mechanisms such as organized religion, family structure, public schools and peer groups [2]. In recent times, the first two mechanisms have lost prominence with the void being filled by the latter two including new media in the internet age. By including "ethics" courses in curricula, institutions of higher learning are also aiming to fill the void and address the on-going need to develop training methods for integrity.

This paper describes the design of the ethics component of a core course at Waterloo ECE290 (Engineering Profession, Law and Ethics). The design marks a departure from traditional approaches to teaching ethics by focusing on personal decision-making processes using the lens of social psychology rather than trying to obtain a desired outcome by employing the tools of moral philosophy. Recently, Benoit Monin and Tamar Schapiro argued in a 2014 Stanford debate [3] that they are skeptical whether teaching students the particulars of various ethical viewpoints encourages them to behave more morally. Monin, who teaches psychology and organizational behavior at Stanford, observed that teaching students social psychology, rather than moral 
philosophy, is potentially a more effective way to inculcate social responsibility.

In ECE290, ethics was framed as being the art of considering the multiple differing perspectives of multiple different stakeholders. Correspondingly, the most ethical decision then is one that takes appreciates the full diversity of perspectives in any given situation and employs a sound decision-making process to resolve the issue. To reinforce the importance of understanding and refining personal decision-making processes, students engaged in discussion on the identity question, understanding master behavioral patterns and group dynamics. The class lectures were supplemented by inclass discussion of various relevant case studies. Later versions of the same course required students to understand behavioral patterns and identities associated with characters in two books: Man's Search for Meaning and All Quiet on the Western Front. Course evaluation data indicates that the behavioral pattern material in particular was widely appreciated and detailed comments indicated that this material connected at a deep personal level with some students.

It must be emphasized that the key difference between the current and more traditional versions of teaching ethics is the focus on the student's decision-making process as opposed to the desire to train and elicit any particular outcome. Though there is a chance the resulting decision may appear unsound and even counter to values held by various stakeholders or even the majority, yet the freedom to take decisions free from coercion (but not free from responsibility or consequences) is a necessary part of living in a free society. Embodying and inculcating such a process for decision-making is paramount because it leads away from moralizing, turns "I should" into "I could", and empowers one to take ownership of actions.

Specific elements of the course design are described followed by a discussion of student assessment methods. Assessment is particularly challenging given the focus of the ethics component has shifted away from the need to produce any desired answer. Lastly, student responses based on course evaluation questionnaires are analyzed.

\section{ETHICS COMPONENT DESIGN}

Although the course ECE290 encompasses material on Engineering Profession, Ethics and Law, effort was expended primarily on the ethics component while the engineering profession and law components followed the more traditional teaching approaches in engineering. In traditional courses on ethics, an ethics theory segment is undertaken to establish an ethical foundation based on popular philosophical theories (e.g. Kant, Locke). They theory is then followed by practical discipline specific case analyses, and sometimes supplemented by in-class debates. In the current approach, the ethical theory part of the course was replaced with class discussions on three topics connected to social psychology: (1) the identity question, (2) master behavioral patterns and (3) group dynamics and group identity.

\subsection{The Identity Question}

The identity question defines and discusses the key motivation for the course right at the outset with the students. The motivation is framed as understanding personal behavior and day-to-day actions in the context of one's identity as a mechanism to draw out and understand different perspectives within the class. For example, students with strong religious identities might respond differently to blasphemy than students from a nonreligious perspective. Alternately, gender differences can give rise to different perspectives on personal relationships. Also, immigrants might have a different perspective on finding employment in contrast to fifth generation Canadians.

Once the link between identity and perspective was established, the course examined how a particular identity (and thus set of perspectives) can be formed. To understand this, elements of social psychology were introduced and in particular, how mental models form due to experiencing specific events during the course of one's life. These models are a consequence of the human ability to find correlations and/or causation in everyday events. In addition, the power of repetition in language as a mechanism for forming or breaking beliefs was highlighted.

The purpose of this weeklong discussion was to underscore that perspectives are a consequence of repeated thought patterns or beliefs and are typically formed without the person being aware of the formation (e.g. during childhood). However, a perspective is not a static unchangeable aspect of personality and if required, a person can dynamically change if a particular perspective does not serve them appropriately. For example, someone who procrastinates (i.e. a procrastinator), through introspection, could discover the beliefs, events or experiences that led them to take on the identity and perspective of a procrastinator and if desirable, enact change.

\subsection{Master Behavioral Patterns}

The second change in the ethics component included a segment on behavioral patterns exhibited by humans that were first described by Carl Jung [4]. Jung elaborated in detail on a few of these patterns (also called archetypes), and noted that these patterns are fluid and have a multitude of variations. More recently, Caroline Myss [5] is one of a growing body of authors who has elaborated on Jung's archetypes and the evocative language she employs is striking and particularly compelling for an 
undergraduate university class. From Jung, Myss synthesized and identified four common patterns: child, victim, prostitute, and saboteur.

The child pattern is associated with beliefs and behaviors ingrained into one's psyche due to events during childhood. It is responsible for how trusting or cynically one might view the world. The victim pattern is associated with blaming external sources or alternately, avoiding personal responsibility for one's actions whereas recognizing the victim pattern in oneself can be very empowering. The prostitute pattern links the impact of incentives (e.g. money) or disincentives (e.g. punishment) on decision-making. And lastly, the saboteur pattern identifies the desire for status quo or alternately, resistance to change.

The purpose of introducing the students to behavioral patterns is to create a sense of self-awareness in everyday situations. Examining one's behavior through Jung's archetypal lens and using Myss' language of patterns can lead to a conclusion that one's identity is not set in stone. The idea is that if one believes that one's identity and hence everyday actions are parts of a wider behavioral pattern (i.e. mind on autopilot), then one is not fated to repeat the past and behavior can potentially be changed in the moment in any situation simply by being aware of when the pattern emerges in oneself.

\subsection{Group Dynamics and Group Identity}

The last segment of the ethics component involved introducing the class to challenges associated with decision making in a group environment. This is a natural second step given the first two parts of the ethics segment were focused on monitoring personal identity, behavior and recognizing patterns in oneself. Using some examples (e.g. Mann Gulch, Bay of Pigs, Cuban Missile Crisis) where group dynamics have been attributed as being instrumental to exacerbating the situation, this segment concludes with a look at the group dynamics aspect of the Challenger Space Shuttle disaster.

Although engineering courses typically focus on the technical fault finding processes and conclude that whistle blowing was essential, fewer authors have examined the role of group dynamics in the Challenger disaster. Referencing material from the book on NASA culture by Diane Vaughn [6], the role of group dynamics is explored highlighting the need to adopt an inquiry mindset in specific situations as opposed to an advocacy mindset. Transcripts from the telephone call between Morton Thiokol and NASA the night before the launch are used to facilitate role play in the class helping emphasize the dysfunction that existed in the group dynamics.

The discussion on group dynamics also naturally brings to light the role of group identity or organizational culture. Group culture is introduced as an extension and interplay of the behavioral patterns of various individuals who are either currently or were part of the history of the organization.

\section{STUDENT ASSESSMENT METHODS}

As noted in the introduction, efforts to teach a set of given values or any kind of basic ethical principles to university students are likely to fail because of the myriad of perspectives on any given topic. Also, learning in a free society cannot willingly force an outcome perceived as desirable by any one set of stakeholders. Evaluation methods in an ethics course (such as an instructor defined "right" answer) are typically perceived as moralistic and patriarchal and serve to demotivate students. Thus, effort was invested into creating assignments and evaluation methods that focused on the process by which students made decisions rather than aiming for a desired outcome. The course work was divided into researching and discussing four cases, analyzing and comparing two books and the midterm and final exams.

\subsection{Book Analyses}

Two books were selected for review purposes: Man's Search for Meaning (MSM) and All Quiet on the Western Front $(A W Q F)$. MSM, written by Austrian psychiatrist, Victor Frankl describes the author's personal experience in a WWII deathcamp, and in particular, his hallmark conclusion that even in the most difficult circumstances, life has potential meaning and that it falls upon the incumbent to find that meaning. MSM also provides many opportunities for students to observe ethically challenging situations (i.e. in the death camps) and the behavioral patterns of the various characters which fulfills three key objectives of the ethics component: the first being to increase the students' skill in recognizing ambiguous situations, the second being to recognize when a character is caught in a behavioral patterns and third to recognize that behavioral patterns can be used in both empowering and disempowering ways.

The second book, AWQF, is fiction authored by a former German war veteran Erich Maria Remarque. AWQF was selected to provide parallels and contrast to MSM. Although both are books about war, AWQF depicts war from the perspective of young German soldiers in WWI, who appear all too similar to their English and French counterparts. Like prisoners in MSM, soldiers in AWQF engage in patterns that are mostly disempowering and like many prisoners, most soldiers feel bitter and disillusioned towards the end of the war. What is striking though is that in both books, there are characters who are able to use their behavioral patterns to empower themselves and this is what is highlighted during class discussions and subsequently discussed by the students during the book review assignment. 


\subsection{Case Studies}

Case studies were chosen to represent a variety of topics that would be of interest to electrical and computer engineering $2^{\text {nd }}$ year undergraduate students. The first case study focused on a product malfunction and how the manufacturer responded to the public outcry. Examples of cases used in the past for this particular topic include Ford Pinto, spontaneously combusting Black \& Decker coffee makers and the ambiguity surrounding thalidomide prescriptions for morning sickness. The second case study delved into individual responsibility, whistle blowing and the associated challenges of group dynamics when working in a large organization. The case used for this topic is Challenger but examined from additional perspectives of group dynamics and group identity as noted earlier. The third case topic focuses on the role of the engineer with the environment. For example, the class analyzed the perspectives of multiple stakeholders and the responsibility of key individuals in power in the Chalillo Dam construction by Fortis and recently, the Keystone oil pipeline. The last case topic involves interplay between ethics and law and grappling with the concept that a decision could be legal but not ethical or vice versa. Here, sample cases include legal challenges brought to bear by Blizzard on the open source developers of bnetd.org and the legal controversy over the use of enhanced interrogation techniques by US law enforcement agencies after 9/11.

Students were placed into groups of 3 or 4 and their submissions were evaluated based on whether the group could identify the ambiguous situations in the case topic, the stakeholders in the situation, the various perspectives that could be held by each stakeholder, how the situation was or could be resolved, and why was (or not) the resolution satisfactory from the student group perspective. As noted, the case evaluations focused on the mechanisms by which student groups analyzed each situation and not on the decision outcome. Thus, the students gained experience on understanding and presenting different perspectives, even if these perspectives were diametrically opposed to their own initial viewpoint.

\subsection{Examinations}

The ethics component on the midterm and final examination consisted primarily of testing whether the students could recognize behavioral patterns in characters found in MSM and/or AWQF or even in prominent characters found in a case assignment. For example, during the Challenger case roleplay and group dynamics dialogue in class, students became intimately familiar with Boisjoly from Morton Thiokol and George Hardy from the Marshall Space flight Center. The students were challenged to recognize in these characters, common patterns like Victim or Prostitute. Lastly, on the midterm examination, a case study on sexual harassment was presented and the students were asked to analyze the material individually using the same approach they had practiced in their group case study assignments.

\section{STUDENT FEEDBACK}

The University of Waterloo course evaluation questionnaire is divided into two sections, characteristics of instructor and characteristics of the course. The characteristics of the instructor include: the ability to present organized lectures, response to questions, oral and visual presentation, approachability, ability to explain material, encouraged to think and reason objectively independently on subject matter, professor's attitude to teaching the course, professor-class relationship and the quality of teaching. The characteristics of the course include: difficulty of concepts, workload, usefulness of textbook, assignments, tests and tutorials, appraisal of course and number of classes attended. Also included is the total number of students providing responses.

Over the last five course offerings by the same instructor, differences were observed in the course evaluation metrics. Some differences observed in the quality of teaching and overall appraisal of the course were correlated with the professor-class relationship which is often qualitatively, a function of course workload, midterm difficulty and perceived fairness in marking.

In Fig. 1, three metrics including encouraged to think independently, difficulty of concepts and appraisal of course are plotted over five different course offerings (from 2012 to 2014). There are a number of notable differences across the offerings. For example, in the first two course offerings, material on identity and behavioral patterns was not included. In the third offering, identity and behavioral patterns including the book review were introduced for the very first time. However, in this third offering, there were two additional assignments on identity that asked students to write about and assess their own identities. Marking these identity assignments proved to be a challenge since any external input on such a deeply personal issue can be perceived as a moral judgment (i.e. a departure from the original course focus on the decision making process towards what a "right" decision or identity must be). This particular issue is likely what led to a poor class-professor relationship in offering \#3, a significant drop in the appraisal of course metric (the lowest the author has ever received in fourteen years of teaching across two different institutions) and a corresponding decrease in all evaluation metrics.

When the identity assignments were subsequently removed in the following two course offerings (\#4 and \#5), the appraisal of course metric increased significantly 
even though the course material on identity and behavioral patterns was retained. Interestingly, introducing the identity and behavioral pattern course material was correlated with a small but observable increase in the difficulty of concepts and encouraged to think metrics between the first two and the last two offerings.

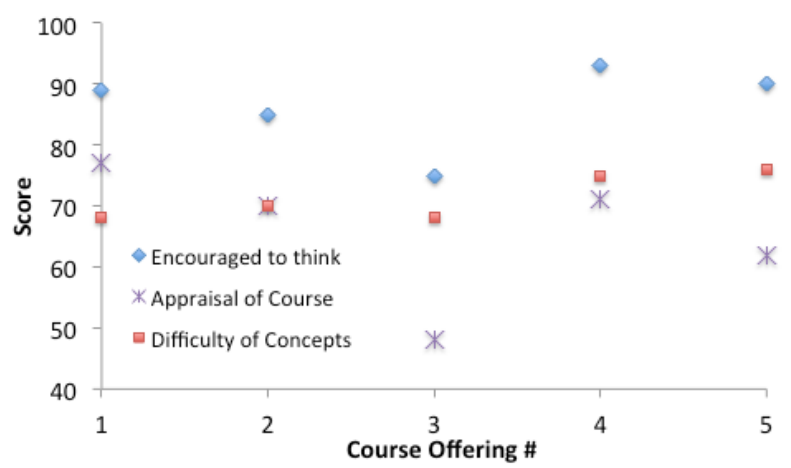

Fig. 1. Key metrics from the Waterloo course evaluation questionnaire

In Fig. 2, the percent of students responding to the course evaluation questionnaire is plotted over five course offerings. Here, course evaluation questionnaires were provided to students to be filled out during a regular class session at least two weeks after the midterm and at least two weeks before the final exam. The date for filling out the questionnaire is not announced in advance and thus, the percent of students responding can be taken as correlated to the typical class attendance. As seen, the percent of students responding across the five offerings ranges from $60 \%$ to $75 \%$ and is on par with technical course offerings in the second year undergraduate engineering program. However, a caveat here is that in the first two offerings, a small daily class participation mark $(2 \%)$ was employed which may have potentially skewed the data for course offering \#1 and \#2.

Lastly, beyond the quantitative components of the Waterloo course feedback form, student engagement can also be gauged by the qualitative written feedback on the course evaluation questionnaire. While the volume of written feedback was similar across all five offerings of the course, some students provided very specific comments about the kind of changes motivated in them after taking the course in the last two course offerings. In the last two course offerings, the vast majority of comments were related to the in-class discussion of behavioral patterns indicating this material in particular resonated strongly with students.

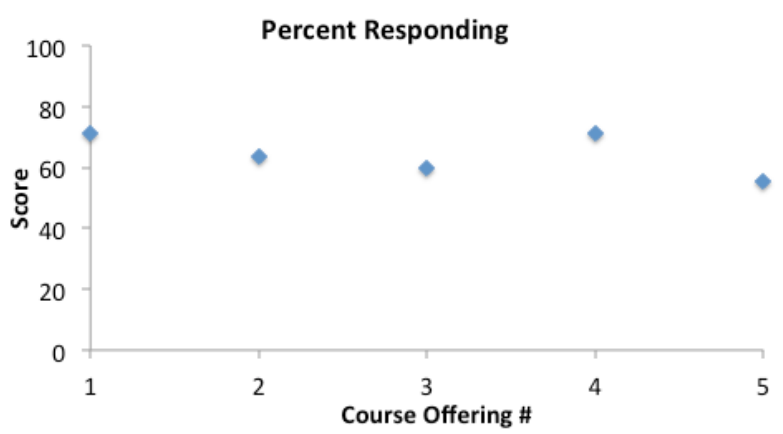

Fig. 2. Percent of students responding to the course evaluation questionnaire

\section{Acknowledgements}

The author gratefully acknowledges Prof. S. Bedi and the Ideas Clinic team at the University of Waterloo for encouragement to prepare and submit this work.

\section{References}

[1] Anne Colby and William M. Sullivan, "Ethics Teaching in Undergraduate Engineering Education," Journal of Engineering Education, vol. 97, no. 3, pp. 327-338, 2008.

[2] Tom C. Clark, "Teaching Professional Ethics", San Diego Law Review, vol. 12, No. 2, pp 249-260, 1975.

[3] Salil Dudani, "Stanford panel debates: Does teaching ethics do any good?," Stanford Report, May 13, 2014. Available as of April 13, 2015 from

http://news.stanford.edu/news/2014/may/ethics-in-society051314.html

[4] Carl Gustav Jung, The Structure and Dynamics of the Psyche (Collected Works of C.G. Jung, Volume 8), edited and translated by G. Adler and R.F.C. Hull. Princeton, NJ: Princeton University Press, 1970.

[5] Caroline M. Myss, Sacred Contracts: Awakening Your Divine Potential. Three Rivers Press, 2003. 442 pages

[6] Diane Vaughan, The Challenger Launch Decision: Risky Technology, Culture, and Deviance at NASA. University Of Chicago Press, 1997. 592 pages 